\title{
Synthesis and Stochastic Assessment of Cost-Optimal Schedules
}

\author{
Angelika Mader $^{1}$, Henrik Bohnenkamp ${ }^{2}$, Yaroslav S. Usenko ${ }^{3}$, \\ David N. Jansen ${ }^{1}$, Johann Hurink ${ }^{1}$, Holger Hermanns ${ }^{4,1}$ \\ 1 University of Twente \\ Faculty EWI, 7500 AE Enschede, The Netherlands \\ e-mail: $\{$ a.h.mader $\mid$ david.n.jansen $\mid j \cdot 1 . h u r i n k\} @ u t w e n t e . n l$ \\ 2 RWTH Aachen University \\ Modeling and Verification of Software Group (MOVES), Info 2, 52056 Aachen, Germany \\ e-mail: henrik@cs.rwth-aachen.de \\ 3 Technical University of Eindhoven \\ Laboratory for Quality Software (LaQuSo), 5600 MB Eindhoven, The Netherlands \\ e-mail: Y.S.Usenko@tue.nl \\ 4 Saarland University \\ Dependable Systems and Software Group, 66041 Saarbrücken, Germany \\ e-mail: hermanns@cs. uni-sb.de
}

\begin{abstract}
We present a novel approach to synthesize good schedules for a class of scheduling problems that is slightly more general than the scheduling problem $F J m, a\left|g p r, r_{j}, d_{j}\right|$ early/tardy. The idea is to prime the schedule synthesizer with stochastic information more meaningful than performance factors with the objective to minimize the expected cost caused by storage or delay. The priming information is obtained by stochastic simulation of the system environment. The generated schedules are assessed again by simulation. The approach is demonstrated by means of a non-trivial scheduling problem from lacquer production. The experimental results show that our approach achieves in all considered scenarios better results than the extended processing times approach.
\end{abstract}

Key words Schedule synthesis - Stochastic - Resource Failures - Heuristics

\section{Introduction}

In this paper we deal with a scheduling problem where the resources are prone to failures. The stochastic nature of machine breakdowns makes the problem hard to tackle and, in practice, they cannot only cause delays that were not anticipated, but can devaluate the whole schedule. Looking at the current literature on scheduling (see e. g., [25] for an overview) one observes that stochastic effects are hardly taken 
into account. Only for simple models (mainly single machine models) methods exists to deal explicitely with stochastic influences on the data. A common way to get rid of the uncertainty is to abstract to expected values and to incorporate slack or margins into the data.

A possible realization of this approach for stochastic machine breakdowns is to define so called performance factors, which describe the ratio $p=$ uptime/ (uptime+ downtime) of a resource. The approach to account for failures then extends the processing times of tasks by a factor of $1 / p$. These extended processing times (EPT) are then used to generate deterministic schedules.

This approach has a justification, if acceptable production load on the long term has to be investigated. A different, relevant question is, however, how this approach works if orders have to be finished at a certain due date. It is possible to generate schedules with extended processing times, which do meet the due date, but the question is to what extent these schedules hold up in a realistic environment, where resources do fail.

We will investigate this problem by means of a case study that was proposed in the context of the meanwhile finished IST project AMETIST (Advanced Methods for Timed Systems) [3]. The case study was suggested by the industrial partner Axxom, a company that provides planning and logistics solutions. This case study is about schedule generation for lacquer production. Lacquers are produced according to certain recipes, which are sub-divided into tasks. The execution of tasks requires resources, which are prone to failures. The failure behaviour is described by performance factors. Three types of recipes are considered: metal, uni, and bronce. Each order is specified by a lacquer type and a due date. In the framework of the case study, a fixed set of 29 orders is used. We use this case study as a vehicle to demonstrate our approach.

In a previous paper [10], we have investigated schedules in the lacquer production framework with respect to feasibility, i.e., whether orders are finished before their due dates. The schedules are synthesized using the model checker UPPAAL [26], both for net and extended processing times. In a real production setting with stochastic elements, deterministic schedules can not be followed completely, in general. Therefore, we focus on the starting times of orders, and regard them as the most relevant element of a schedule. We assess the schedules by simulation, making reasonable assumptions about the failure behaviour of resources. We create a simulation model of the lacquer production plant using the modelling language MODEST [12], and simulate the 29 orders according to the generated schedule with the simulator of the performance tool MöBIUS $[16,18,11]$. The quintessence of this study is that schedules generated with extended processing time have more chance to miss the due date than schedules based on net processing times. This can be explained by observing that in the EPT approach time is reserved for machine failure, even in the case the machine is up. If the machine goes down later, not enough time is left to repair it and finish in time.

The original question of the case study as proposed by Axxom, however, was not focussing on feasibility, but on schedules that minimize an objective function. Following these lines we include costs for each order missing its due date. There are two types of cost: storage costs, which are incurred when an order is finished 
before the due date, and delay costs, which are incurred if an order is finished after the due date. We consider again the scenario of 29 orders as described before, with the difference that every order has not only a due date, but also a storage cost factor and a delay cost factor attached. As before, resources are prone to failure.

Our research question here is how to synthesize schedules that minimize costs in a setting where resources can fail. More precisely, we suggest a new approach and evaluate it against the EPT appraoch. Technically, we synthesize different sets of schedules, for one part following the EPT approach, for the other part following our suggested method, and assess all by stochastic simulation.

The first set of schedules we generate is based on net and EPT processing times, respectively. The tool we use is the model-checker UPPAAL CORA, a version of UPPAAL which allows to attach costs to states of the considered model and which allows to find schedules that minimize the costs. The principles of this approach have been described in several publications $[8,5,6]$.

The second set of schedules is generated according to our new approach. We proceed in two steps: first, for each individual order we determine a quantity which we call the optimal starting time (OST). Second, we synthesize schedules where the effective starting times of orders are as close as possible to their respective OSTs.

OSTs are determined in a way that starting an order at its OST minimizes the expected cost. Note that the OSTs are not chosen such that the mean completion time of an order is its due date, which constitutes the main difference to the EPT approach. We derive the OSTs by simulating the different recipes and determine the run-time distribution for each order type. UPPAAL CORA is used to generate schedules with effective starting times of orders as close as possible to the respective OSTs. Due to resource conflicts orders might not be able to start at their precomputed OSTs. The synthesized schedule contains information about starting times of all tasks and orders, but we regard the release times of orders in the production process as the only relevant information.

As in [10], we assess the quality of the schedules by simulation, making reasonable, more detailed assumptions about the failure behaviour of the resources. Our measure of interest, for the EPT- as well as for the OST-based schedules, is the mean total cost that is incurred by executing all 29 orders according to the schedule.

Our experiments show that the OST based schedules indeed effectuate less expected costs than the EPT based schedules. Schedules based on net processing times give highest costs. Additionally, it turns out that our approach is also robust against erroneous assumptions on the failure distribution: even if the failure distribution as basis for the OSTs differs from the failure distribution chosen in the simulation, the cost is not higher than for the EPT approach, and in most cases significantly better. Apart from the result that our suggested approach works, the experiments also show that there is room for more fine-tuning of the OSTs, which gives rise to future research.

While we demonstrate our approach on this particular example, it is worth to highlight, that it generalizes to a whole range of settings. Furthermore, the tools we use, UPPAAL CORA and MÖBIUS could, in principle, be substituted by other 
tools. For us, the flexibility in modelling different settings, and the capability to deal with our questions, made them comfortable to use.

Organisation of the paper: The lacquer-production case study we use to demonstrate our approach is introduced in Section 2. In Section 3, we present the tools to generate and simulate schedules. In Section 4 we explain our OST approach in detail. Experiments and their results are collected in Section 5. We conclude with Section 6.

\section{Lacquer Production Case}

The purpose of this section is to present the necessary details of the lacqer-production case study, which has a non-trivial scheduling problem with several complex aspects. Research on this case-study has led to several publications [10,24,5,6].

We start with a short description of the case and continue with its classification following the standard scheme for machine scheduling problems [19].

There are three recipes for different types of lacquer: metallic, bronce and uni lacquers. Each recipe describes the processing steps, their durations, their interdependency, precedence relations with timing constraints, and finally, the resources to use. A recipe is composed of tasks. Each task describes which resource it requires, and for how long that resource is needed. The latter time value is called the processing time of the task. Figure 1 contains a graphical representation of the three considered recipes. The rectangular objects represent the tasks. The patterns indicate the resource type needed to execute the task, and the numbers the processing time in minutes. The tasks related to mixing vessels are implicit, since they do not have prespecified processing times but last as long as the parallel tasks are performed. Vertical lines denote dependencies between the tasks (top to bottom), and horizontal lines represent synchronisation. In the case no numbers are given next to the vertical lines, the dependencies express ordinary precedence constraints between the tasks, indicating that the later task may only start its processing after the former task has finished. If an interval $[a, b]$ is given next to the vertical line, this indicates that the later task has to start later than the preceding task by an amount in range between $a$ and $b$.

An order of a certain type of lacquer is specified by the type of lacquer to be produced and the earliest possible starting time and the due date until which it has to be finished. An order that is finished before the due date will cause storage costs, finishing after the due date causes delay costs. Axxom assumes that costs are incurred linearly with time, i.e., the storage cost and delay cost can be described by a piecewise linear function with two parameters $s c f$ and $d c f$ : the storage cost factor and the delay cost factor. As a result, the costs $c(d, t)$ of finishing a job with due date $d$ at time $t$ is given by

$$
c(d, t)=\left\{\begin{array}{l}
s c f(d-t) \text { if } t \leq d \\
d c f(t-d) \text { if } t>d .
\end{array}\right.
$$

Figure 2 contains a schematic representation of the cost function with respect to the due date. An order finished precisely at the due date will cause zero costs. 


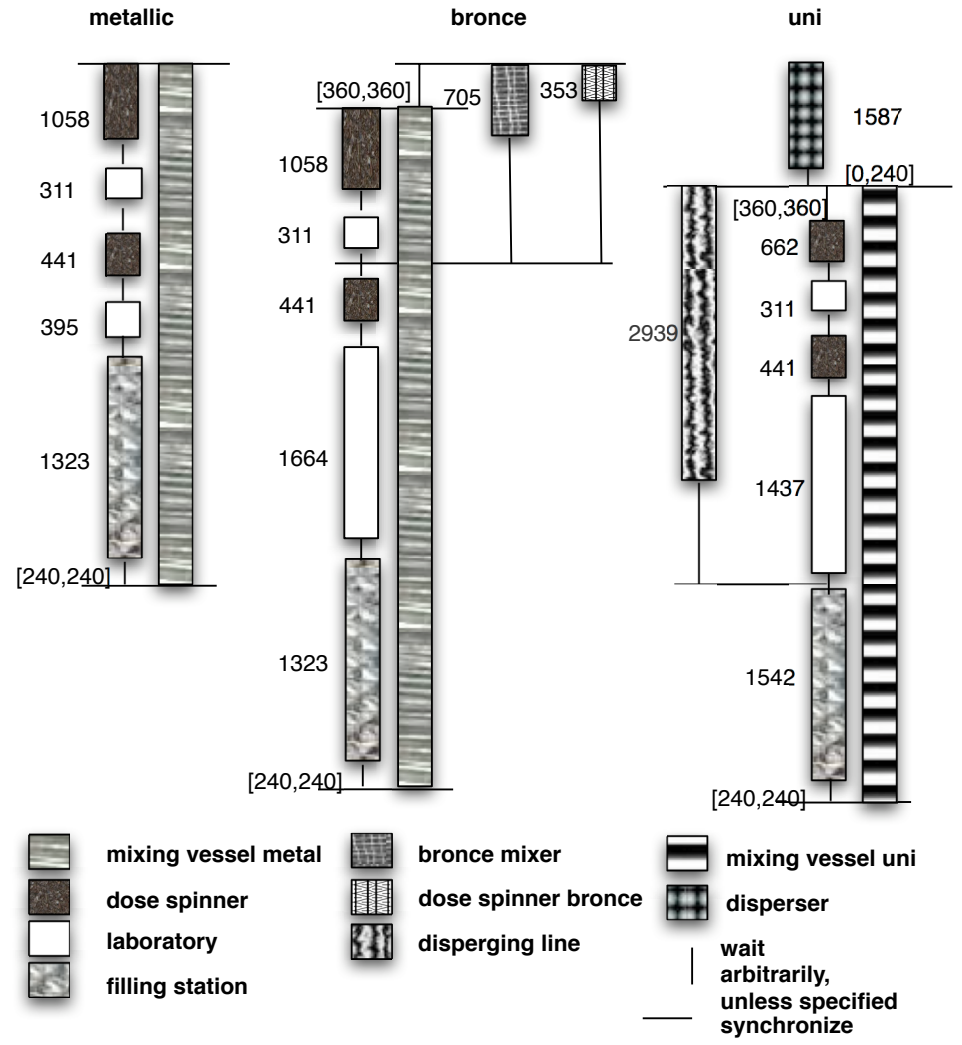

Fig. 1 Lacquer recipes indicating the resources needed, durations of processing steps and precedence relations with timing constraints

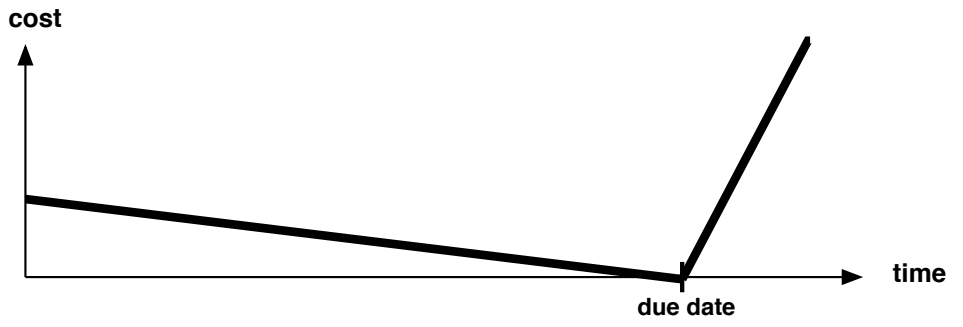

Fig. 2 Cost function for product finish time 


\begin{tabular}{|r|c|c|r|r|}
\hline order & type & due & $s c f$ & $d c f$ \\
\hline \hline 1 & metal & 27444 & 8 & 429 \\
\hline 2 & metal & 33348 & 8 & 390 \\
\hline 3 & metal & 33636 & 11 & 574 \\
\hline 4 & metal & 36372 & 8 & 425 \\
\hline 5 & metal & 37092 & 10 & 496 \\
\hline 6 & metal & 40500 & 11 & 584 \\
\hline 7 & metal & 41699 & 15 & 779 \\
\hline 8 & metal & 47172 & 10 & 531 \\
\hline 9 & metal & 56964 & 10 & 492 \\
\hline 10 & metal & 57252 & 14 & 708 \\
\hline 11 & metal & 57539 & 14 & 691 \\
\hline 12 & metal & 62148 & 7 & 368 \\
\hline 13 & metal & 67764 & 6 & 283 \\
\hline 14 & metal & 67980 & 7 & 354 \\
\hline 15 & metal & 71868 & 4 & 213 \\
\hline
\end{tabular}

\begin{tabular}{|r|c|c|r|r|}
\hline order & type & due & $s c f$ & $d c f$ \\
\hline \hline 16 & bronce & 43500 & 17 & 9 \\
\hline 17 & bronce & 50580 & 15 & 744 \\
\hline 18 & bronce & 60660 & 10 & 531 \\
\hline 19 & bronce & 61859 & 13 & 673 \\
\hline 20 & bronce & 65100 & 8 & 4 \\
\hline 21 & uni & 30459 & 3 & 177 \\
\hline 22 & uni & 41628 & 3 & 2 \\
\hline 23 & uni & 43764 & 7 & 354 \\
\hline 24 & uni & 44004 & 6 & 283 \\
\hline 25 & uni & 50580 & 10 & 531 \\
\hline 26 & uni & 60588 & 7 & 4 \\
\hline 27 & uni & 61788 & 10 & 5 \\
\hline 28 & uni & 77124 & 8 & 390 \\
\hline 29 & uni & 85764 & 8 & 425 \\
\hline
\end{tabular}

Table 1 The parameters for the 29 considered orders

In the case study, a fixed set of 29 orders is given, each described by a lacquer type, a due date, and respective $s c f$ and $d c f$. In Table 1, we summarize the parameters for the different orders. Note that for most orders, $s c f<d c f$, and the relation $d c f / s c f$ is in the order of 30 to 60 . This observation will become important in Section 4. However, for some few orders, scf $>d c f$ holds.

As can be seen in Figure 1, there are nine different types of resources. Resources are subject to failure, and the failure behaviour of resource type $r$ is described by a performance factor $p_{r}$. Intuitively, the performance factor is the ratio of uptime/(uptime + downtime), where uptime and downtime are the up- and downtime of the resource. The values uptime and downtime must actually be considered as random variables, since the failure of a resource is a stochastic phenomenon. However, no information was available about the mean durations of upand downtime, or even the distributions.

To get a clear understanding of the problem, we classify the lacquer production scenario in the project scheduling terminology (see e.g., [19]). The considered problem is a slight generalization of the problem FJm, a $\left|g p r, r_{j}, d_{j}\right|$ early/tardy. Here

- ' $\alpha=F J m, a$ ' specifies the resource characteristics as a flexible job-shop problem, where

- each order has to follow its own route,

- a fixed number of $m$ resource types are given with a given number of instances per type. In the concrete case 9 resource types with the following instances per resource types are given: 


\begin{tabular}{|c|l|l|l|}
\hline$p_{r}$ & \multicolumn{1}{|c|}{0.85} & \multicolumn{1}{|c|}{0.75} & \multicolumn{1}{c|}{1.0} \\
\hline \hline & & Bronce Mixer & \\
& Dose Spinner & Dose Spinner Bronce & Mixing Vessel Uni \\
$r$ & Laboratory & Disperging Line & Mixing Vessel Metal \\
& Filling Station & Disperser & \\
& & & \\
\hline
\end{tabular}

Table 2 Performance factors for different resource types

$\begin{array}{ll}\text { mixing vessel metal } & 3 \\ \text { mixing vessel uni } & 2 \\ \text { dose spinner } & 2 \\ \text { filling line } & 2 \\ \text { bronce mixer } & 1 \\ \text { dose spinner bronce } & 1 \\ \text { disperging line } & 1 \\ \text { disperser } & 1 \\ \text { laboratory } & \text { unlimited }\end{array}$

- the availability of the resources is stochastic, caused by machine breakdown, and specified as performance factor per resource type. (In Table 2, the performance factors for the concrete case are given. Note that we assume mixing vessels to be failure-free.)

- ' $\beta=g p r, r_{j}, d_{j}$ ' indicates that the task/order characteristics are:

- no preemption of tasks is allowed;

- generalized precedence relations are present;

- deterministic release dates have to be respected;

- deterministic due dates are imposed.

- and ' $\gamma=$ early/tardy' expresses that the performance measure is to minimize the weighted earliness-tardiness of the orders, i.e., orders finished before the due date cause storage costs and orders finished after the due date cause delay cost.

The lacquer production problem is in that sense a bit more general than the above problem, as the resource environment is not exactly a job-shop. The orders do not follow a distinct route but at some points the task chain splits or merges (i.e., the task network of a recipe is not a simple chain but a fork-join extension of a chain).

\section{The tool chain}

For our approach to derive and assess schedules we us two tools. Schedules are synthesized with the model-checking tool UPPAAL CORA [15], an extension of UPPAAL $[26,7,4]$. Simulations are executed with the performance evaluation tool MÖBIUS [18]. Simulation models are specified in the MODEST language [17,12]. 


\subsection{UPPAAL and UPPAAL CORA}

Automata are a well-known description model for concurrent systems. Timed automata [1] are an extension of finite-state automata with real time clocks. Clocks can be reset, used in guards on transitions and in invariants on locations.

UPPAAL is an integrated tool environment for modeling, validation and model checking of real-time systems modelled as networks of timed automata, extended with data types (bounded integers, arrays, etc.). The tool was developed in collaboration between the Department of Information Technology at Uppsala University, Sweden and the Department of Computer Science at Aalborg University in Denmark.

Model checking [14,22] is an advanced method to formally verify concurrent finite-state systems. Specifications about the system are expressed as temporal logic formulas, and efficient symbolic algorithms are used to traverse the model defined by the system and check if the specification holds or not. Extremely large state-spaces can often be traversed within minutes.

UPPAAL CORA [15] is a branch of UPPAAL for Cost Optimal Reachability Analysis. Whereas UPPAAL supports model checking of timed automata, UPPAAL CORA uses an extension of timed automata called linear priced timed automata (LPTA) [9,2]. LPTA allows to annotate the model with the notion of cost. Costs can be defined for delay while staying in a location, or costs for a particular action. UPPAAL CORA then finds optimal paths matching goal conditions.

Scheduling synthesis by model checking works along the following lines: Assume we have a model of the uncontrolled production process. The model checker searches for a state with the property all orders are finished. Once it has found such a state the model checker can provide a diagnostic trace, i.e., a trace from the initial state to the state with the desired property. This trace contains all the infomation on start and finish (times) of production steps, as well as information about resources, and, in the context of scheduling, represents a valid schedule.

This technique can also be applied to linear priced timed automata. Here, the model checker searches for the cheapest state having the desired property. Having found such a state, the diagnostic trace then provides a cost-optimal schedule. In practice we have to deal with very large state spaces and we can not expect to find a cheapest state, resp. an optimal schedule. However, it was shown that this technique, extended by suitable heuristics, allows to find good schedules in very short time $[5,6]$.

What makes the timed automaton environment attractive for solving scheduling problems is the robustness against variations in the parameter setting. By this we mean that timed automata are a general description language and allow for an enormous variation in problem descriptions: it is easy to change, add, or remove model parameters without changing the search mechanism. On the other hand, this advantage comes for the price of state-space explosion, which, however, can be handled by suitable heuristics. 


\subsection{MODEST and MÖBIUS}

MODEST is a formal language to describe stochastic timed systems [17,12], equipped with a rigid formal semantics. The functional core of MODEST can be considered as a simple process algebra enriched with some convenient language constructs. The syntax resembles that of the programming language $\mathrm{C}$ and the modeling language Promela [21]. Data modularization concepts and exception handling mechanisms have been adopted from modern object-oriented programming languages such as Java. Process algebraic constructs have been strongly influenced by FSP (Finite State Processes [23]), a simple, elegant calculus that is aimed at educational purposes.

This core language is enriched with several modeling concepts tailored to model timed and stochastic systems. We highlight three particular semantic concepts which are well-established in the context of real-time and stochastic discrete event systems:

- Probabilistic branching is a way to include quantitative information about the likelihood of choice alternatives.

- Clocks are a means to represent real time and to specify the dynamics of a model in relation to a certain time or time interval, represented by a specific value of a clock.

- Random variables are often used to give quantitative information about the likelihood of a certain event to happen after or within a certain time interval.

The MODEST language allows one to specify processes, and to compose them in parallel using a parallel composition operator. Processes can manipulate data variables by assignments. Data variables are typed and must be declared, and the point of declaration determines their scope. In particular, they may be local to a process, or global, in which case they are shared between all processes. A particular type of variable which can be declared is the clock type. Clocks can be read like an ordinary float variable, but advance their value linearly to the real-valued simulation time. All clocks run at the same speed. Clocks can only be set to zero. The language provides generic constructs to sample values from a set of predefined probability distributions. For instance, ' $x d=\operatorname{Uniform}(10,20)$ ' assigns a sample from the uniform distribution on the interval $[10,20]$ to the variable ' $x d$ '. Other types of distributions are, e.g., Exponential(rate) and Normal(mean,var).

Apart from manipulating data, processes can interact with other parallel processes (or the environment) by means of actions. Their occurrence within a process can be guarded by a 'when' clause, specifying an enabledness condition. In particular, the boolean expression in a 'when' clause may refer to clock values. In that case, an action may be enabled as soon as the when condition becomes true (and no other action becomes enabled earlier). We assume a maximal progress semantics, which means that an actions is taken as soon as it is enabled, i.e., idling has lower priority that performing actions. 
MöвIUS is a performance evaluation tool environment ${ }^{1}$. MÖвIUS supports multiple input formalisms and several evaluation approaches for these models. Atomic models are specified in one of the available input formalisms. Atomic models can be composed by means of state-variable sharing, yielding so-called composed models. Notably, atomic models specified in different formalisms can be composed in this way. This allows to specify different aspects of a system under evaluation in the most suitable formalism. Along with an atomic or composed model, the user specifies a reward model, which defines a reward structure on the overall model. Note that rewards are the vehicle to define the costs for our case study.

On top of a reward model, the tool provides support to define experiment series, called Studies, in which the user defines the set of input parameters for which the composed model should be evaluated. Each combination of input parameters defines a so-called experiment. Before analyzing the model experiments, a solution method has to be selected: MӧвIUS offers a powerful (distributed) discrete-event simulator, and, for Markovian models, explicit state-space generators and numerical solution algorithms. It is possible to analyze transient and steady-state reward models. The solver solves each experiment as specified in the Study. Results can be administered by means of a database.

MOTOR. In order to facilitate the analysis of MODEST models, we have developed the prototype tool MOTOR [13]. The philosophy behind MOTOR is to connect MODEST to existing tools, rather than re-implementing existing analysis algorithms anew. This requires a well-designed interfacing structure of MOTOR, which is described in [13].

The integration of MODEST into MÖBIUS is done by means of MOTOR. From a user-perspective, the MÖвIUS atomic model interface to design MODEST specifications is an ordinary text editor. Due to the possibility to specify non-Markov and non-homogeneous stochastic processes, only simulation is currently supported as a suitable evaluation approach for MODEST models within M ÖBIUS. While it is in principle possible to identify sublanguages of MODEST corresponding to Markov chain models, this has not been implemented in MOTOR yet.

\section{The Approach}

In this section we present the technical aspects of the OST approach in more detail. In Section 4.1, we motivate why to use OSTs to steer the schedule synthesis with UPPAAL CORA, and how to derive the OSTs. In Section 4.2, we describe our approach to derive completion-time distributions for recipes, in order to derive OSTs. In subsection 4.3 we describe the timed automata models that form the basis for schedule synthesis with UPPAAL CORA.

\footnotetext{
1 The Möbius software was developed by W.H. Sanders and the Performability Engineering Research Group (PERFORM) at the University of Illinois at Urbana-Champaign. See http://www.mobius.uiuc.edu/.
} 


\subsection{Optimal Starting Times}

The state-of-the art approach to solve scheduling problems in the presence of failures is extending processing times according to performance factors, which describe the failure behaviour of resources. The problem with this approach is that schedules based on this principle are actually not only scheduling resources, but, implicitly, also downtimes of resources. This is an adequate approach only in special cases, in particular, if the time to failure of resources and the respective repair times are likey to be short with low variance, compared to the length of processing times of tasks.

In our OST approach, we tackle the problem on a different level: instead of considering the processing times of individual tasks, we consider complete recipes. Due to the failure of resources, the run-time of an order is not of fixed length, but is rather of stochastic nature, which can ideally be described by a distribution function. The stochastic nature of resource failures makes it impossible to find a schedule that will always finish the order precisely at the due date. With some probability, the order is finished too early (in case that time has been reserved for the order, anticipating failure which did not occur), or too late (in case that the repair of resources took longer than anticipated). In both cases, costs are incurred. The case where an order will indeed finish in exactly the amount of time it was planned will be rare.

This observation indicates that it is fruitless to try and make every order costneutral. However, assuming the existence of a distribution function describing the run-time of an order makes it possible to reason about the expected costs incurred by an order. Our approach is based on the goal to minimize the expected costs incurred by all orders. In the following, we will describe this approach in detail.

In a first step, we assume that we have given a distribution function $G(t)$ with density $g(t)$ describing the processing time $T$ of an order. We want to find an optimal offset $d_{o p t}$, by which the order should start before its due date to minimize the mean cost incurrent by the order. For a cost function $c(d, t)$ given by the values $d c f$ and $s c f$, the mean costs for an offset $\bar{d}$ is given by the integral

$$
\int_{0}^{\infty} c(\bar{d}, t) \cdot g(t) d t
$$

Thus, to calculate $d_{\text {opt }}$, we have to look for the zeros of

$$
\begin{aligned}
& \frac{d}{d x} \int_{0}^{\infty} c(x, t) \cdot g(t) d t \\
= & \frac{d}{d x}\left(\int_{0}^{x} s c f(x-t) \cdot g(t) d t+\int_{x}^{\infty} d c f(t-x) \cdot g(t) d t\right) .
\end{aligned}
$$

In fact, we can derive the following property to characterize $d_{\text {opt }}$ :

$$
\frac{G\left(d_{o p t}\right)}{1-G\left(d_{o p t}\right)}=\frac{d c f}{s c f} \text {. }
$$

In the case that $G(t)$ is strictly increasing, $d_{\text {opt }}$ is unique. 
It is straightforward to derive $d_{\text {opt }}$ from $G(t)$, especially if $G(t)$ is described by an discrete approximation. In the next section we show how we derive such an discrete approximation by means of simulation.

\subsection{Estimating Order Completion-Time Distributions with MODEST and MÖBIUS}

Our principal approach is to derive an estimation of the distribution $G(t)$ by simulation. In a nutshell, we have modelled the recipe for each order type in MODEST, and simulated the model in MöBIUS. To obtain meaningful results, it is however also necessary to model faithfully the environment in which an order usually runs. There are two main influences on the execution time of an order: first, the possible failures of resources, and second, the influence of other orders running in parallel.

As far as the failures of resources go, we modeled the breakdown and repair behaviour of all resources by means of two distributions, describing the time-tofailure, and the time-to-repair, respectively. In Section 5 we will describe in detail which distributions and which parameters for the distributions we have chosen.

To account for the mutual influence of orders running in parallel on the completion time of the order we are interested in requires to create a reasonable background load in the simulation model. In essence, we created the following load model:

Parallelism: The description of the case study limited the number of orders that can be executed in parallel to five. We thus make sure in the model that actually five orders are running.

Starting of orders: We start five orders at random times, where the starting times are drawn according to a uniform distribution over an interval of length 720 minutes (12 hours). The completion time of one of these orders is measured.

Type of orders: The type of the orders (metal, bronce, or uni), are chosen at random, except for the one that is measured.

In our experiments that we will describe in Section 5, we have carried out one million simulation runs, producing a histogram ranging from 0 to 20000 minutes with a step size of 10 minutes.

\subsection{UPPAAL Models}

In this section we want to sketch the timed automaton models used for scheduling. The models used here build on earlier work $[5,6]$.

To represent the uncontrolled production process we have a timed automaton for each order. More precisely, each of the three recipes is modelled as a timed automaton with parameters (template), and instantiated these templates with earliest starting time and due date, which defines the orders.

In an automaton (template) processing steps are represented by locations. We defined clocks, invariants on clocks, and clock guards to determine the duration of time spent in a location (production step), and timing constraints between production steps. Resources are modelled as global variables, i.e. counters. For resources 
with more complex structure it would also be possible to model these as automata, but this was not necessary in our case. Availability of a resource at a moment can therefore be checked by reading the counter associated to this resource.

We identified states which are "too late", i.e., where the order is still processed although the due date has already passed, and assigned delay costs to them. Similarly, storage costs are assigned to states, where the order is already finished, but the due date has not been reached.

In order to reduce the state space it is necessary to add some heuristics. In earlier work $[5,6]$ we experimented with several heuristics. In the current context, we used only greediness as strategy and restrict the number of orders that may be processed at any point in time. Not restricting the number of "active" orders would lead to more resource conflicts and unefficient backtracking in the search tree. Moreover, the precise search strategy that we followed is best cost random depth first, meaning that among all direct successors of the current state having the same best cost, one successor is selected randomly for further search.

First, we generated two sets of schedules, one based on net processing times, the other one based on the extended processing times. For both we used the model with cost annotation as defined in the case description: storage and delay costs defined for each order individually.

Second, we generated schedules based on the OSTs. Here, we had to follow a different strategy: the objective was to find schedules with starting time as close as possible to the OSTs given. Taking simply the OSTs as starting times is not enough: there are still resource conflicts that have to be solved, and these may require to start some orders earlier than the OST would suggest. We defined a metric that reflects as close as possible to the optimal starting time. The intuition of this metric is that for orders with higher storage than delay costs, starting after the optimal starting time will be punished less later, and for orders with higher delay than storage costs, starting earlier will be punished less. Therefore we simply shifted the cost definition for the completion time to the optimal starting time. The costs for the schedules synthesized now indicate how "good" the optimal starting times are approximated.

\section{Experiments}

\subsection{Assumptions on Failure Behaviour}

In order to experiment with our approach to derive cost-optimal starting times ( $c f$. Section 4.2), we need to make several assumptions on the failure behaviour of the resources. In this section, we define four different sets of parameters which we use for our experiments, and describe the reasoning behind our choices.

In the original case description, the failure behaviour of the resources is described only by the performance factor $p_{r}=$ uptime/(uptime+downtime) (cf. Section 2). The performace factor itself is stochastically quite meaningless, since it does not give any indication on the durations of up- and downtimes. Thus, we have to make assumptions on these times. As a first approach to relate up- and 


\begin{tabular}{|l||c|c|c|}
\hline Scenario & uptime & downtime & pace \\
\hline \hline$E 100$ & Exponential & Exponential & $100 \mathrm{~h}$ \\
\hline$E 31$ & Exponential & Exponential & $31.6 \mathrm{~h}$ \\
\hline WU100 & Weibull & Uniform & $100 \mathrm{~h}$ \\
\hline WU31 & Weibull & Uniform & $31.6 \mathrm{~h}$ \\
\hline
\end{tabular}

Table 3 The considered failure behaviour of resources

downtimes to durations, we introduce the so called pace parameter, which we define as $p a c e=E[$ uptime + downtime $]$. We then define $E$ [uptime $]=p_{r}$ pace and $E[$ downtime $]=\left(1-p_{r}\right)$ pace. The pace, together with the performance factors, gives information about the failure rate and the mean time between failure. The higher the pace, the higher the mean time to failure and the higher the mean repair time. For our study, we chose two different values of paces: 100 hours, and $10 \sqrt{10} \approx 31.6$ hours.

For the purpose of simulation, it is necessary to describe uptime and downtime by means of distributions. The natural choice here is the exponential distribution: it is solely characterised by one parameter, the mean value, and is the only distribution with this property, and is therefore the choice with the maximal entropy ( $c f$. , e.g., [20]).

In order to see how far the distributions of uptime and downtime influence our results, we consider a second set of distributions to use in our experiments. The very common distribution to describe times to failures is the Weibull distribution. A Weibull distribution is related to the exponential distribution, however, where the exponential distribution has a constant rate, the Weibull distribution has a variable rate, depending on one of its parameters. We chose deliberately a Weibull failure distribution where the failure rate of the component increases polynomially with degree 1.8 over time. This parameter is the only deliberate choice we make here. The other parameters of the Weibull distribution are determined by the previous assumptions on the pace and the performance factors. Together with the Weibull distribution for the time to failure, we choose for the time to repair an uniform distribution, which again is determined wholly by the pace and performance factors.

In comparison, the time to failure is in the exponential case more random than in the weibull case: the squared coefficient of variation of an exponential distribution is 1 , whereas for the Weibull distribution, as we use it here, the coefficient is 0.33 . Therefore, the failure of a resource is more "predictable" than in the exponential case.

In summary, we consider in our study four different scenarios, depending on the choice of paces and distributions, as shown in Table 3. We name the four scenarios E100,E31, WU100, and WU31 throughout the rest of the paper.

\subsection{Generation of Optimal Starting Times}

As described in Section 4.1, it is necessary to derive the completion time distribution for each recipe. We have done this for the four scenarios described in 5.1 

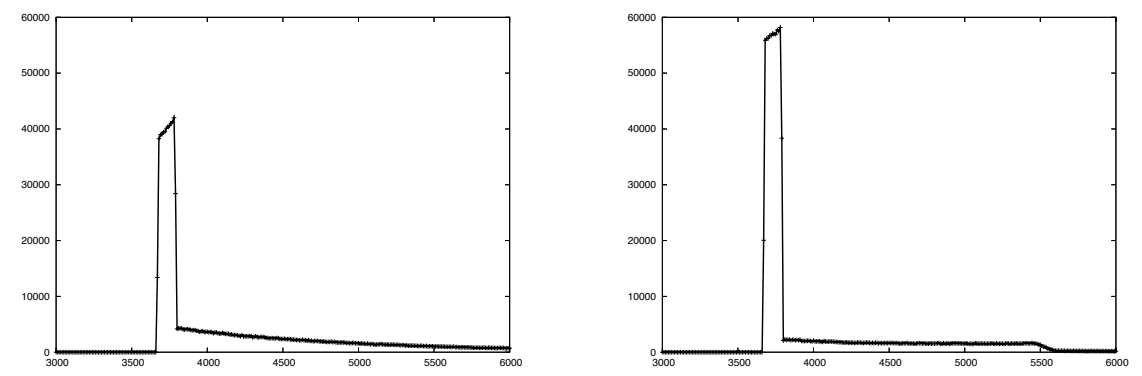

Fig. 3 Histogram for the cases E100 and WU100 for job type metal

\begin{tabular}{|r|c|c|}
\hline Order & Offset & OST \\
\hline \hline 1 & 7810 & 19634 \\
\hline 2 & 7700 & 25648 \\
\hline 3 & 7780 & 25856 \\
\hline 4 & 7800 & 28572 \\
\hline 5 & 7720 & 29372 \\
\hline 6 & 7800 & 32700 \\
\hline 7 & 7770 & 33929 \\
\hline 8 & 7800 & 39372 \\
\hline
\end{tabular}

\begin{tabular}{|r|c|c|}
\hline Order & Offset & OST \\
\hline \hline 9 & 7710 & 49254 \\
\hline 10 & 7740 & 49512 \\
\hline 11 & 7710 & 49829 \\
\hline 12 & 7790 & 54358 \\
\hline 13 & 7660 & 60104 \\
\hline 14 & 7740 & 60240 \\
\hline 15 & 7800 & 64068 \\
\hline
\end{tabular}

Table 4 Optimal offsets and starting times for metal orders for E100 in minutes

by means of simulation. In Figure 3 we see the histogram for metal orders in the case of E100 and WU100. Using Equation (1), it is straightforward to determine how long before its due date an order should be started. We call this the optimal offset of the order. We derive thus for each order an optimal offset. Subtracting these offset from the due date of the respective order yields the optimal starting time. In Table 4, we give the optimal offset and the OST for the case E100 and job type metal. The recipe of job type metal in Figure 1 indicates that the minimal time that a metal order needs to complete is 5037 minutes (the length of the critical path). As we can see in the table, the optimal offsets derived by our approach are in the range of 7660 to 7810 minutes, i.e., about 2700 minutes (ca. 2 days) earlier. These 2700 minutes, approximately $35 \%$ of the whole time, is thus reserved for potential breakdowns of resources. In Table 5, we see the offsets and OSTs for uni orders. The uni recipe in Figure 1 shows that the minimal completion time of a uni job is about 6580 minutes (about 4.5 days). In Table 5, the optimal offsets are quite diverse. For orders $21,23-25$, and 28-29, the offsets are in the order of 19000 minutes $(\approx 13$ days $)$-nearly three times the minimal time. On the other hand, for orders 22 , and 26-27, the optimal offset lies in the area of 8000 minutes, which is only $20 \%$ longer than the minimal time. The reason for that can be found in Table 1: for orders 22 and 26-27, scf $>d c f$, which means that, if the due date is to be missed, it is preferable to be late, rather than early. Apparently, the optimal offsets for the respective orders are taking this into account. 


\begin{tabular}{|r|r|r|}
\hline Order & Offset & OST \\
\hline \hline 21 & 19390 & 30459 \\
\hline 22 & 8320 & 41628 \\
\hline 23 & 18920 & 43764 \\
\hline 24 & 18710 & 44004 \\
\hline 25 & 19070 & 50580 \\
\hline
\end{tabular}

\begin{tabular}{|r|r|r|}
\hline Order & Offset & OST \\
\hline \hline 26 & 8110 & 60588 \\
\hline 27 & 7930 & 61788 \\
\hline 28 & 18810 & 77124 \\
\hline 29 & 19070 & 85764 \\
\hline
\end{tabular}

Table 5 Optimal offsets and starting times for uni orders for $E 100$ in minutes

\subsection{Generation of schedules}

Using UPPAAL CORA, we produced 6 sets of schedules:

- 10 Schedules, using netto processing times. We call this class S.NPT.

- 10 Schedules, using extended processing times. We call this class S.EPT.

- Four classes of schedules with 10 schedules each, taking the optimal starting times of the four scenarios E100, E31, WU100, and WU31 into account ( $c f$. Section 5.1). We call these four classes of schedules S.E100, S.E31, S.WU100, and $S . W U 31$, respectively.

The objective function for the S.NPT and S.EPT schedules was to meet the due dates as good as possible, causing costs for storage and delay as in the case description.

For the four classes S.E100, S.E31, S.WU100, and S.WU31, the objective function for the schedule synthesis was to find schedules that have the starting times of orders as close as possible to the OSTs and that have resolved the resource conflicts.

As explained in Section 3.1, the search strategy we use is best cost random depth first. Experiments show that this search strategy gives the best results: the state space is too big to be traversed completely. Due to the randomness and the size of the search space we got schedules of different quality, i.e., with high cost variations. In order to generate a set of ten schedules we always performed a much higher number of schedules, depending on the model the number of experiments is a factor of 3-10 higher. A single experiment, however, takes less than a minute in most cases, less than 3 minutes in all cases.

The most efficient heuristic used is the limitation of the number of orders that are processed at each moment. The upper bound we chose is 5, and was found after a number of experiments. It is plausible, considering the bottleneck resources: the dose spinners, that are available twice, but have to be used by every order, the disperging line, and to some extent also the mixing vessels. Moreover, we chose a greedy strategy for resource distribution. If several orders are waiting for a resource, the the one who gets the resource is chosen nondeterministically, i.e. we do not have a waiting queue model. Heuristics reducing the time windows for starting orders, were also applied. Much effort went to this fine-tuning, which is justified by the goal to find schedules as close as possible to the OSTs. 


\subsection{Analysis by simulation}

As explained in Section 4, our method to assess the quality of deterministic schedules is stochastic simulation. In this section we will present the results of the simulations.

In this experiment, we simulated seven classes of schedules. The first six classes are those generated with UPPAAL CORA, as described in the previous section. The seventh class is a set of four schedules, based on the OST only. Since we take in our approach only the starting times of orders into account, it is possible to regard the optimal starting times already as schedule as well: we start each order exactly at its optimal starting time. Thus, for each of the four schedule classes S.E100, S.E31, SWU100, and S.WU31 we have one extra schedule, defined by the OSTs for that class. We call the respective schedule S.OST.E100, S.OST.E31, S.OST.WU100, and S.OST.WU31.

Our measure of interest is, for each schedule, the mean total cost that accumulates during the execution of all 29 orders. We simulated all schedules of all considered classes. The mean value is estimated with a relative confidence interval $<10 \%$ and a confidence level of $99 \%$.

The schedules in class S.NPT and S.EPT were simulated for all the scenarios E100,E31, WU100, and WU31. The schedules S.E100, S.E31, SWU100, and S.WU31 were each only simulated with the corresponding scenario, i.e., S.E100 with $E 100$, etc.

In order to reduce the size of the tables with the results, we do not present the outcome for all schedules per class, but rather the average $(E[\cdot])$ and relative standard deviation (coefficient of variation $c_{v}$ ) of the respective outcomes. The numeric results are summarised in Table 6 .

\subsection{Interpretation of Simulation Results}

5.5.1 Comparison of the S.NPT and S.EPTschedules. The average costs of the S.NPT schedules range from 8 Mio. to 12 Mio., depending on the chosen scenario. The relative standard deviation ranges from ca. 12 to $17 \%$. This shows that, even though all schedules in this class resolve resource conflicts, the way it is done has an influence on the costs that are incurred.

The average costs of the S.NPT schedules are the highest in the whole table. This is not surprising, since these schedules do not take the failure of resource into account at all. Therefore, for each order the probability to overshoot the due date by a considerable amount of time is very high. The S.NPT numbers show how bad costs can become.

The average cost of schedules in the S.EPT class range from ca. 2.5 Mio. to 6 Mio., depending on the chosen failure scenario. The relative standard deviation is between $17 \%$ and $38 \%$. The S.EPT schedules are better than the S.NPT schedules. We explain this by the fact that more time is calculated for the execution of each task, and thus for each order. The numbers suggest that S.EPT can indeed help saving considerable costs, compared to the S.NPT schedules. 
Failure model used for schedule analysis

\begin{tabular}{|c|c|c|c|c|c|}
\hline Schedule set & Measure & $E 100$ & $E 31$ & WU100 & WU31 \\
\hline 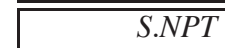 & $\overline{E E[\cdot]}$ & $\overline{12.131 .001}$ & 9.628 .586 & 9.720 .134 & 8.568 .726 \\
\hline & $c_{v}$ & $11.88 \%$ & $15.51 \%$ & $14.16 \%$ & $16.97 \%$ \\
\hline S.EPT & $E[\cdot]$ & 5.907 .007 & 3.340 .511 & 3.800 .883 & 2.530 .444 \\
\hline 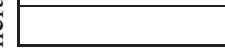 & $c_{v}$ & $17.17 \%$ & $29.28 \%$ & $26 \%$ & $37.12 \%$ \\
\hline S.E100 & $E[\cdot]$ & 1.683 .969 & & & \\
\hline & $c_{v}$ & $1.93 \%$ & & & \\
\hline S.E31 & $\overline{E[\cdot]}$ & & 855.446 & & \\
\hline & $c_{v}$ & & $3.76 \%$ & & \\
\hline S.WU100 & $E[\cdot]$ & & & 1.313 .474 & \\
\hline & $c_{v}$ & & & $4.79 \%$ & \\
\hline S.WU31 & $\overline{E[\cdot]}$ & & & & 785.304 \\
\hline & $c_{v}$ & & & & $7.47 \%$ \\
\hline S.OST.E100 & & 1.732 .719 & & & \\
\hline S.OST.E31 & & & 1.445 .109 & & \\
\hline S.OST.WU100 & & & & 2.209 .187 & \\
\hline S.OST.WU31 & & & & & 2.165 .868 \\
\hline
\end{tabular}

Table 6 Results for total cost for different schedules

The variation in the costs for the S.NPT and S.EPT schedules confirm that there are good schedules and bad schedules. At this point we are not able to determine a priori, what schedule is a good one without simulation.

5.5.2 Comparison of the S.EPT and the S.E/S.WU schedules. We consider first the S.E100 case. Table 6 shows, that the average costs incurred by these schedules is about 1.7 Mio. The relative standard deviation is with $1.93 \%$ very low. We can see, that the average cost of the S.E100 schedules is only $28.5 \%$ of the average cost of the S.EPT schedules for the E100 scenario. Since the variation of the S.EPT schedules is in the order of $17 \%$, we can assume a best-case schedule which is indeed $17 \%$ better than the average. On the other hand, we can assume that there is a S.E100 schedule that is $1.93 \%$ worse than the average. Setting best-case and worstcase schedules in relation, the costs of the worst-case S.E100 schedule would still be only $35 \%$ of the best-case S.EPT schedule.

For the $S . E 31$ schedules, the average costs are about 0.86 Mio., with a relative standard deviation of $3.8 \%$. This is $25.6 \%$ of the costs of the S.EPT schedules in the E31 scenario. Assuming again best-case/worst-case schedules, the S.E31 schedule would still cost only $38 \%$ of the S.EPT schedule.

The S.WU100 schedules reduce the costs to $34.5 \%$ of the S.EPT schedules, and, assuming the best-case/worst-case again, the cost is still reduced to about $49 \%$.

Finally, the S.WU31 cost on average only $31 \%$ of the S.EPT schedules, and in the extreme case, still only $53 \%$. 
These results lead us to the main conclusion of this paper: OST schedules are in general cheaper than EPT schedules, and thus better.

5.5.3 Comparison of S.E/S.WU and S.OST.E/S.OST.WU schedules. The schedules in the $S . E / S . W U$ classes have been generated with UPPAAL CORA. Since the optimal starting times can also be seen as schedules themselves, the question arises whether UPPAAL CORA is needed at all, and if these S.OST.E/S.OST.WU schedules are not actually sufficient.

The numbers in Table 6 show that this is in general not the case. Even though for the S.E100/S.OST.E100, the costs are very close (around 1.7 Mio.), for the other cases the differences become more pronounced: the UPPAAL schedules generate $60 \%$, in the WU31 case even only $36 \%$ of the costs of the corresponding OST schedules.

Our explanation for that is that the schedules generated with UPPAAL CORA do resolve resource conflicts by letting orders start earlier (if $s c f<d c f$ ), or later (if $s c f>d c f$ ). Thus, the effect is that the probability to complete the order on the expensive side of the cost function is reduced. Consequently, the step of producing refined OST with UPPAAL CORA is indeed necessary to reduce the mean total costs further.

\subsection{Robustness of the OST approach}

The results of the previous section indicate that our approach to use OST schedules to reduce the mean total costs of a batch of orders is indeed a promising one. In this section we show that even if we make inaccurate assumptions on the failure distributions, and thus work with inaccurate OSTs to generate schedules, in all cases the costs are not higher than for the EPT approach, and in most cases significantly better. In order to find out how stable our approach is, we have sim-

\begin{tabular}{|r||r|r|r|r|}
\hline & $E 100$ & $E 31$ & WU100 & WU31 \\
\hline \hline S.EPT & 5.907 .007 & 3.340 .511 & 3.800 .883 & 2.530 .444 \\
\hline \hline S.E100 & $\mathbf{1 . 6 8 3 . 9 6 9}$ & 1.105 .239 & 1.139 .555 & 1.079 .691 \\
\hline S.E31 & 2.232 .010 & $\mathbf{8 5 5 . 4 4 6}$ & 986.859 & 702.696 \\
\hline S.WU100 & 3.047 .240 & 1.070 .889 & $\mathbf{1 . 3 1 3 . 4 7 4}$ & 684.322 \\
\hline S.WU31 & 3.842 .751 & 1.416 .179 & 1.769 .831 & $\mathbf{7 8 5 . 3 0 4}$ \\
\hline
\end{tabular}

Table 7 Average mean total costs for all OST schedule with all scenarios

ulated all S.E/S.WU schedules also with the parameters for which the schedules where not generated for. We would expect that the simulations with the "proper" parameters should always give lower costs than the others. This is however not always the case. In Table 7, we see the averages for all combinations of schedule classes and failure scenarios. For comparison, we have also repeated the values for the S.EPT schedules from Table 6. Also the values set in boldface come from 
the same Table. For the case of scenario E100 we see that the schedules in class S.E100 produce minimal cost: the non-diagonal values in the respective column are all much higher. The same holds still for the case E31: the schedules of class S.E31 are the lowest in the column.

The situation is different for the $W U$ scenarios. In both cases, the corresponding $S . W U$ schedules do not yield the lowest cost. In case of WU100 it would be better to use a schedule from the S.E31 class. In case of WU31, it would be better to use one from $S$.WU100.

The cause of this phenomenon is subject to further research. Nevertheless, even though wrong assumptions on distributions might give not the best results, a comparison with the S.EPT results shows that our OST approach produces still substantially better results.

\section{Conclusions}

In this article, we present an alternative to the EPT approach to generate schedules that take the possible failures of resources into account. The pivotal elements of our approach are the so-called optimal start times of orders. These OSTs are estimated by means of simulation and straightforward mathematical derivations. They reflect release times of orders for which the expected cost incurred by the orders are minimal. We use the OSTs as input to our schedule synthesizer, the model-checker UPPAAL CORA.

We assess the properties of all generated schedules by means of stochastic simulation. The simulation models, described in the modeling language MODEST, are simulated with MÖBIUS. The simulation models comprise resources prone to failure, the recipes, and the schedule in question. We have considered four different cases of failure behaviour for the estimation of OSTs, generation of schedules and the simulation of schedules.

The simulation results show that the schedules that we have derived with the OST approach are in all four considered scenarios substantially cheaper than the schedules derived with the EPT approach. Our approach is robust in the sense that, even if assumptions on the failure behaviour of resources are inaccurate, the OST schedules still incur lower costs then their EPT counterparts.

Our main conclusion is using optimal starting times for the generation of schedules to be a favourable alternative to the EPT approach.

However, it is also clear that our approach could be improved by more fine tuning. Relevant questions are what the right assumptions for derivation of the OSTs are, e.g., what the best estimates for distribution of resource failure are, and what the best assumptions on the background load of a system. Then, taking OSTs as a basis for derivation of schedules, the question is what is a schedule that approximates the OSTs best. We suggested a cost function with an obvious intuition, but possibly there are better strategies. Another direction could be to be more explicit over the load in a system. There are moments with low load, and others where the load is high. Our OSTs are all based on high load, for moments with low load they are too pessimistic. We could calculate different OSTs for different loads and 
apply the best fitting OST during scheduling when it is clear how many concurrent orders have to be processed in the close future.

Also, the scheduling strategy we followed in both schedule synthesis and simulation is greedy, and when there are several tasks waiting for a resource, one task is chosen nondeterministically that gets the resource. Possibly other strategies, such as activeness (non-laziness), non-overtaking, or waiting-queues could give better results.

The tools we used, UPPAAL CORA and MÖBIUS have the necessary modelling and processing capabilities that are needed for the demonstration of our approach. However, these tools do not yet have industrial strength. Modelling effort that, in principle, can be automated was done by hand here, which is a time-consuming process. For an industrial application, modelling should be mechanized and performed with tool-support. Nevertheless, UPPAAL CORA and MÖBIUS are under continuous improvement, aiming at industrial applicability.

Acknowledgements We give our thanks to Ed Brinksma for discussion on the approach, and to Martijn Hendriks and Gerd Behrmann, who helped us with models and tools.

\section{References}

1. Rajeev Alur and David L. Dill. A theory of timed automata. Theoretical Computer Science, 126(2):183-235, 1994.

2. Rajeev Alur, Salvatore La Torre, and George J. Pappas. Optimal paths in weighted timed automata. In Maria Domenica Di Benedetto and Alberto SangiovanniVincentelli, editors, Hybrid Systems: Computation and Control; ... HSCC, volume 2034 of $L N C S$, Berlin, 2001. Springer.

3. AMETIST, IST project ist-2001-35304. http://ametist.cs.utwente.nl/.

4. Tobias Amnell, Gerd Behrmann, Johan Bengtsson, Pedro R. D’Argenio, Alexandre David, Ansgar Fehnker, Thomas Hune, Bertrand Jeannet, Kim G. Larsen, M. Oliver Möller, Paul Pettersson, Carsten Weise, and Wang Yi. UPPAAL: Now, next, and future. In Franck Cassez, Claude Jard, Brigitte Rozoy, and Mark Dermot Ryan, editors, Modeling and Verification of Parallel Processes: 4th summer school, MOVEP 2000, volume 2067 of $L N C S$, pages 99-124, Berlin, 2001. Springer.

5. Gerd Behrmann, Ed Brinksma, Martijn Hendriks, and Angelika Mader. Production scheduling by reachability analysis: A case study. In 19th IEEE International Parallel and Distributed Processing Symposium: Workshop on Parallel and Distributed RealTime Systems; WPDRTS, page paper 140a, Los Alamitos, California, April 2005. IEEE Computer Society.

6. Gerd Behrmann, Ed Brinksma, Martijn Hendriks, and Angelika Mader. Scheduling lacquer production by reachability analysis: a case study. In P. Horacek, M. Simandl, and P. Zitek, editors, Preprints of the 16th IFAC World Congress: Prague [DVD], pages Mo-A17-TO/3, Laxenburg, Austria, July 2005. International Federation of Automatic Control.

7. Gerd Behrmann, Alexandre David, and Kim G. Larsen. A tutorial on UPPAAL. In Marco Bernardo and Flavio Corradini, editors, Formal Methods for the Design of Real-Time Systems: ... SFM-RT 2004, number 3185 in LNCS, pages 200-236, Berlin, September 2004. Springer. 
8. Gerd Behrmann, Ansgar Fehnker, Thomas Hune, Kim Larsen, Paul Petterson, Judi Romijn, and Frits Vaandrager. Minimum-cost reachability for priced timed automata. In Maria Domenica Di Benedetto and Alberto Sangiovanni-Vincentelli, editors, Hybrid Systems: Computation and Control; ... HSCC, volume 2034 of LNCS, pages 147-161, Berlin, 2001. Springer.

9. Gerd Behrmann, Ansgar Fehnker, Thomas Hune, Kim G. Larsen, Paul Petterson, and Judi Romijn. Guiding and cost-optimality in UPPAAL. In Lina Khatib and Charles Pecheur, editors, Model-Based Validation of Intelligence: Papers from 2001 AAAI Spring Symposium. AAAI, 2001.

10. H. C. Bohnenkamp, H. Hermanns, R. Klaren, A. Mader, and Y. S. Usenko. Synthesis and stochastic assessment of schedules for lacquer production. In First International Conference on the Quantitiative Evaluation of Systems: QEST, pages 28-37, Los Alamitos, CA, September 2004. IEEE Computer Society.

11. Henrik Bohnenkamp, Tod Courtney, David Daly, Salem Derisavi, Holger Hermanns, Joost-Pieter Katoen, Vinh Vi Lam, and William H. Sanders. On integrating the MöBIUS and Modest modeling tools. In 2003 International Conference on Dependable Systems and Networks: ... DSN, page 671, Los Alamitos, CA, 2003. IEEE Computer Society.

12. Henrik Bohnenkamp, Pedro R. D'Argenio, Holger Hermanns, and Joost-Pieter Katoen. MoDeST: A compositional modeling formalism for hard and softly timed systems. Technical Report TR-CTIT-04-46, Centre for Telematics and Information Technology, University of Twente, Enschede, The Netherlands, November 2004.

13. Henrik Bohnenkamp, Holger Hermanns, Joost-Pieter Katoen, and Ric Klaren. The ModEST modeling tool and its implementation. In Peter Kemper and William H. Sanders, editors, Computer Performance Evaluation: ... TOOLS, volume 2794 of LNCS, pages 116-133, Berlin, 2003. Springer.

14. Edmund M. Clarke, Jr., Orna Grumberg, and Doron A. Peled. Model Checking. MIT Press, Cambridge, MA, 1999.

15. UPPAAL CORA home page. www.cs.aau.dk/ behrmann/cora/.

16. David Daly, Daniel D. Deavours, Jay M. Doyle, Patrick G. Webster, and William H. Sanders. Möbius: An extensible tool for performance and dependability modeling. In Boudewijn R. Haverkort, Henrik C. Bohnenkamp, and Connie U. Smith, editors, Computer Performance Evaluation: ... TOOLS, volume 1786 of LNCS, pages 332336, Berlin, 2000. Springer. Tool Description.

17. Pedro R. D'Argenio, Holger Hermanns, Joost-Pieter Katoen, and Ric Klaren. MoDeST: A modelling and description language for stochastic timed systems. In Luca de Alfaro and Stephen Gilmore, editors, Process Algebra and Probabilistic Methods: ... PAPM-PROBMIV, volume 2165 of LNCS, pages 87-104, Berlin, 2001. Springer.

18. Daniel D. Deavours, Graham Clark, Tod Courtney, David Daly, Salem Derasavi, Jay M. Doyle, William H. Sanders, and Patrick G. Webster. The Möbius framework and its implementation. IEEE Transactions on Software Engineering, 28(10):956-969, 2002.

19. Erik L. Demeulemeester and Willy S. Herroelen. Project Scheduling: A Research Handbook. Kluwer, Boston, 2002.

20. Peter G. Harrison and Naresh M. Patel. Performance Modelling of Communication Networks and Computer Architectures. Addison-Wesley, Wokingham, 1993.

21. Gerald J. Holzmann. The SPIN model checker: primer and reference manual. AddisonWesley, Boston, 2004.

22. Michael Huth and Mark Ryan. Logic in Computer Science: Modelling and reasoning about systems. Cambridge University Press, Cambridge, 2000.

23. Jeff Magee and Jeff Kramer. Concurrency: State Models and Java Programs. Wiley, Chichester, 1999. 
24. Sebastian Panek, Sebastian Engell, and Cathrin Lessner. Scheduling of a pipeless multi-product batch plant using mixed-integer programming combined with heuristics. In Luis Puigjaner and Antonio Espuña, editors, European Symposium on Computer Aided Process Engineering 15: ... ESCAPE-15, volume 20 A/B of Computer-aided chemical engineering, pages 1033-1038, Amsterdam, 2005. Elsevier.

25. Michael Pinedo. Scheduling: Theory, Algorithms, and Systems. Prentice Hall, Upper Saddle, NJ, 2nd edition, 2002.

26. UPPAAL home page. http: //www . uppaal.com. 